\title{
Inflammatory arthritis mimicking Complex Regional Pain Syndrome (CRPS) in a child: A case report
}

\author{
Zeliha Egilmez, ${ }^{1}$ Selin Turan Turgut, ${ }^{2}$ Afitap Icagasioglu, ${ }^{1}$ Irem Bicakci ${ }^{1}$ \\ ${ }^{1}$ Department of Physical Medicine and Rehabilitation, Istanbul Medeniyet University, Goztepe Training and Research Hospital, \\ Istanbul, Turkey \\ ${ }^{2}$ Physical Medicine and Rehabilitation Clinics, Red Crescent Society Private Commodity Exchange Hospital, Konya, Turkey
}

\begin{abstract}
Joint complaints in childhood are seen frequently and differential diagnosis can be difficult. Juvenile idiopathic arthritis (JIA) is the most common rheumatological disease of childhood. It involves peripheral joint arthritis, chronic synovitis, and extra-articular manifestations. Accurate diagnosis can take a long time and sometimes multiple diagnoses are used while following the patient until a final diagnosis can be reached. Arthritis may be triggered by trauma and confused with other diseases like complex regional pain syndrome (CRPS), in which trauma plays a role in the etiology. In the present case, ankle pain in an 8-year-old girl was misdiagnosed as CRPS.
\end{abstract}

Keywords: Arthritis; child; complex regional pain syndrome.

Toin

oint complaints in childhood are common and differential diagnosis can be difficult. Juvenile idiopathic arthritis (JIA) is the most frequently seen rheumatic disease in childhood [1]. JIA is a disease primarily involving the peripheral joints and is accompanied by chronic synovitis and extraarticular findings. Although the etiology and risk factors of the disease are not precisely known, immunogenetic susceptibility and environmental factors are suspected. Infections, physical trauma and stress to joints, and immune hyperactivity against self-antigens may be other causes [2, 3]. Post- traumatic oligoarthritis may be attributed to new autoantibodies produced as a result of damage. The most common type of juvenile rheumatoid arthritis (JRA) is oligoarthritis accompanied by antinuclear antibody (ANA) positivity and uveitis, which is more commonly observed in girls [4]. JIA is diagnosed clinically. The precise diagnosis may take a long time. Therefore, patients may be monitored using multiple diagnoses at the outset.

CRPS is a syndrome characterized by pain in distal extremities, edema, restricted mobility, skin changes and vasomotor disorders. It is classified 
as CRPS Type 1 (reflex sympathetic dystrophy [RSD]), which develops without a known nerve lesion, and CRPS Type 2 (causalgia), which develops due to a nerve lesion [5]. Various symptoms and findings, such as allodynia, hyperalgesia, swelling, sweating and changes in skin blood flow, motor weakness, increased or decreased hair growth, thin and glowing skin, and osteoporosis may indicate CRPS [6]. The most common cause is trauma, resulting in bone fracture and soft tissue damage. However, there is no underlying cause in $2-17 \%$ of cases. This article presents a JIA case that was mistaken for CRPS Type 1.

\section{CASE REPORT}

An 8-year old girl presented at the clinic with complaints of pain, swelling and restricted mobility persisting for 3 months in her right ankle (Figure 1). She stated that she had initially sought emergency medical attention for pain in her ankle after unintentionally stepping on her foot. Two-sided ankle radiography appeared normal, and naproxen sodium of $20 \mathrm{mg} / \mathrm{kg} /$ day, ice therapy, and resting splint were recommended with diagnosis of soft tissue injury. Ankle radiography was repeated after patient presented at orthopedic clinic after 1 month of continued pain, and spotted osteoporosis (OP) was detected in calcaneus and navicular bones (Figure 2). Multiple hyperintense signal increases were observed in spotted form on T2weighted MRI (magnetic resonance imaging) series in millimetric sizes in all tarsal bones, as well

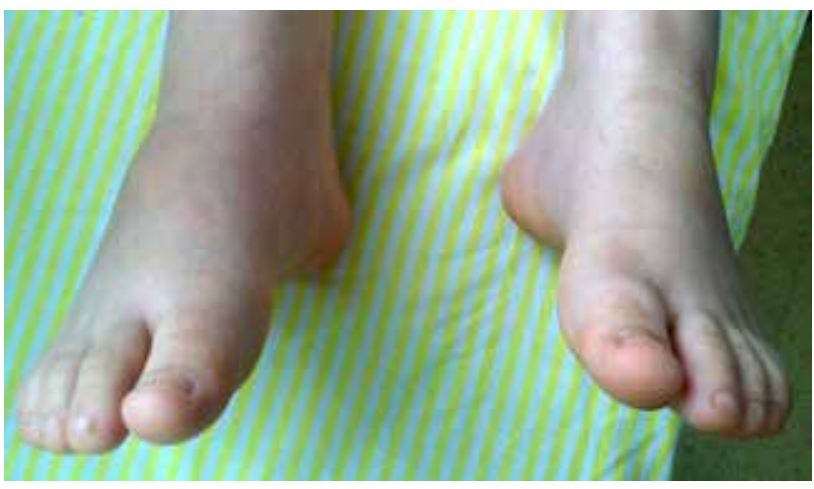

FIGURE 1. Swollen right ankle.

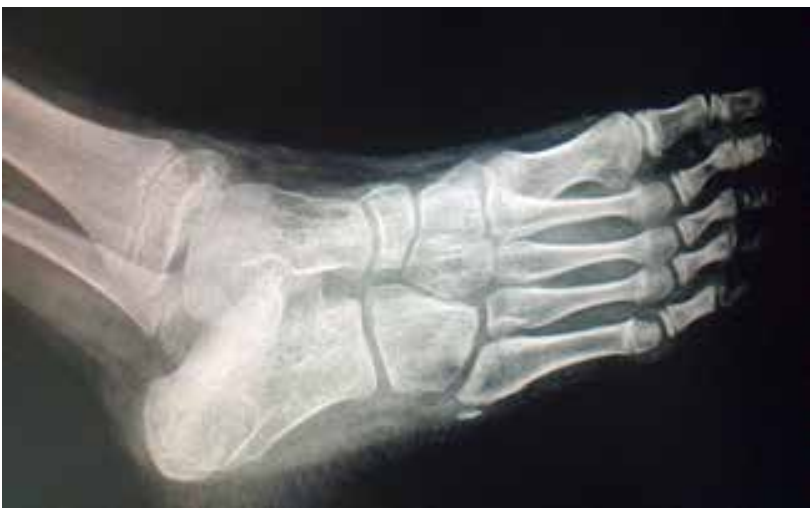

FIGURE 2. Spotted osteoporosis in calcaneus and navicular bones.

as the presence of tibiotalar-diffused intra-articular effusion, fluid in most of the intertarsal joints and surrounding peroneal tendons and tibialis posterior tendon consistent with tendinitis (Figures 3, 4). Diagnosed with CRPS Type 1 secondary to trauma, analgesic treatment and contrast bath were recommended. Patient next presented at outpatient clinic with non-regressed complaints. There was morning stiffness for about 5 to 10 minutes and she limped throughout the day. There were no symptoms of fever, rash, abdominal

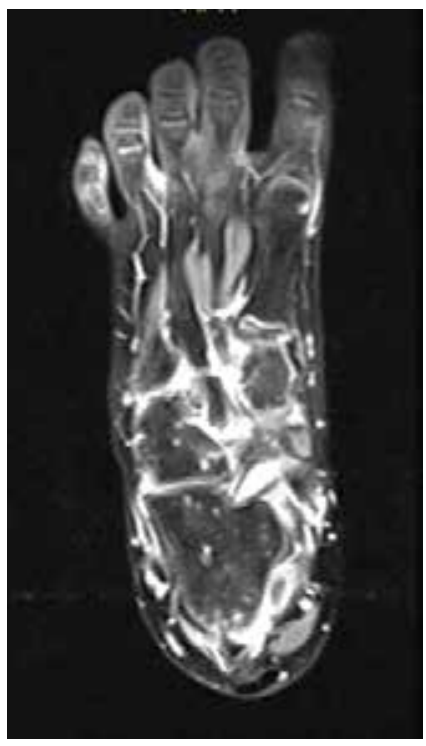

FIGURE 3. Spotted form and millimetric sizes in all tarsal bones, fluid collection surrounding the peroneal tendons and tibialis posterior tendon. 


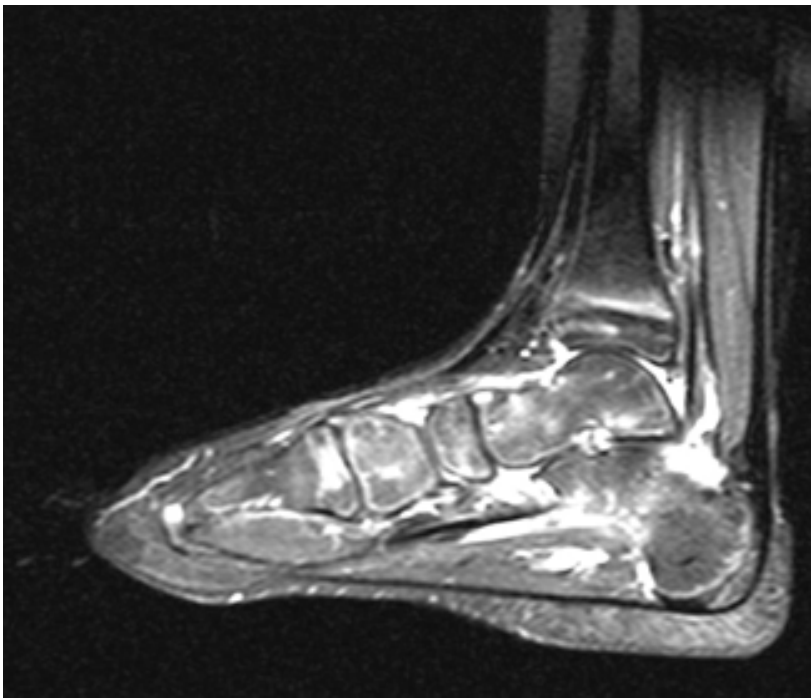

FIGURE 4. Tibiotalar-diffused intra-articular effusion, fluid collection in intertarsal joints.

pain, chest pain, weight loss or muscle weakness. There were no systemic diseases, recent upper respiratory tract infection or chronic drug use in her medical history. There were no specific diseases in family history or systemic examination. Pain that increased with movement, swelling, temperature increase and edema were observed in the right ankle. There was no redness, coldness, hyperhidrosis, hypohidrosis, atrophy, change to hair, allodynia or hyperalgesia. Right ankle mobility ranges were $5^{\circ}$ for dorsiflexion and $45^{\circ}$ for plantar flexion. Examination results for other extremities were normal.

Laboratory results for erythrocyte sedimentation rate (ESR), white blood cell (WBC), hemoglobin and platelet count were found to be 40 $\mathrm{mm} / \mathrm{h}, 13400,12.4 \mathrm{~g} / \mathrm{dL}$ and 334000 , respectively; and renal and hepatic functions, electrolytes and uric acid results were normal. Urine analysis revealed no pathology. Anti-streptolysin-O (ASO), $\mathrm{C}$-reactive protein (CRP), rheumatoid factor (RF) and anti-double-stranded DNA antibody (antidsDNA) results were negative. While immunoglobulin (Ig) A, Ig G, and Ig M levels were normal, Ig E was $671.00(\uparrow)$. Anti-nuclear antibody ANA (+) indirect immunofluorescence (IIF) $1 / 100$ Ig G (+) showed a speckled, finely granular pattern. Sacroiliac joint radiography and MRI were normal. Pediatric rheumatology department was consulted with assumed diagnosis of oligoarticular JIA. Pre-diagnosis was confirmed by pediatric rheumatology department, and treatment with sulfasalazine $500 \mathrm{mg} 2 \times 1$ was initiated, as an insufficient response had been obtained with longterm, full-dose nonsteroidal anti-inflammatory drug (NSAID) therapy. Decreases in pain and swelling were observed in a follow-up examination conducted 1 month later, and decreases in inflammatory markers were also observed in laboratory tests (erythrocyte sedimentation rate [ESR]: 11 $\mathrm{mm} / \mathrm{h}, \mathrm{WBC}: 8700)$.

\section{DISCUSSION}

JIA defines a group of diseases characterized by arthritis of unknown origin that lasts for more than 6 weeks in 1 or more joints with onset before 16 years of age. It is classified as oligoarticular, polyarticular, or systemic-onset JIA based on clinical findings and laboratory characteristics within first 6 months. The most common type of JIA is oligoarticular JIA (50-60\%). Some $50 \%$ of patients present monoarthritis with asymmetric involvement of joints of the lower extremities [7].

In this case, history of trauma, pain, swelling, edema and spotted OP image (spotted OP is not specific to CRPS; it is observed in $30-80 \%$ of cases) suggested CRPS; however, absence of vasomotor/trophic changes, allodynia, hyperalgesia, presence of nonpitting edema and limitation to one joint led the authors to consider laboratory findings in differential diagnosis. Absence of fever, absence of severe pain, sensitivity and erythema in joint, and moderate level of leukocytosis excluded septic arthritis.

Radiological findings of early JIA include OP around the joint, increased intra-articular fluid, joint space widening and periarticular soft tissue edema [8]. Observed osteoporosis must not be considered only for CRPS. Patient complaints that persisted for 3 months (criterion is minimum 6 weeks), increased sedimentation, mild leukocytosis, increased Ig, and positive ANA led to consideration of inflammatory pathologies. Involve- 
ment of single joint, gender and ANA positivity suggested oligoarticular JRA.

NSAIDs are used as first-line therapy for JIA, and NSAID may be administered alone if disease activity is low. In a case with active arthritis, the use of NSAIDs alone for more than 2 months is not recommended $[9,10]$ and a second-line therapy, such as adding sulfasalazine $50 \mathrm{mg} / \mathrm{kg} /$ day is suggested. The present patient responded to treatment with sulfasalazine in addition to NSAID, and the response supported the diagnosis.

In the pediatric population, patients presenting with joint complaints must be evaluated in terms of rheumatologic diseases. Arthritis may be triggered by trauma and confused with other diseases, such as CRPS, where trauma plays a role in etiology. Although there have been CRPS cases that were confused with inflammatory arthritis in literature, we found no JIA case that was confused with CRPS [11]. In conclusion, all pathologies involving locomotor system must be considered in children presenting with joint complaints.

Conflict of Interest: None declared.

Financial Disclosure: The authors declared that this study has received no financial support.

Authorship contributions: Concept - S.T.T.; Design - S.T.T.; Supervision - A.I; Data collection \&/or processing - Z.E.; Analysis and/or interpretation - I.B.; Literature search - Z.E.; Writing - Z.E., S.T.T., I.B.; Critical review - A.I.

\section{REFERENCES}

1. Ravelli A, Martini A. Juvenile idiopathic arthritis. Lancet 2007;369:767-78. Crossref

2. Aslan M, Kasapcopur O, Yasar H, Polat E, Saribas S, Cakan H, et al. Do infections trigger juvenile idiopathic arthritis? Rheumatol Int 2011;31:215-20. Crossref

3. Huang JL. New advances in juvenile idiopathic arthritis. Chang Gung Med J 2012;35:1-14. Crossref

4. Gori S, Broglia AM, Ravelli A, Aramini L, Di Fuccia G, Nicola $\mathrm{CA}$, et al. Frequency and complications of chronic iridocyclitis in ANA-positive pauciarticular juvenile chronic arthritis. Int Ophthalmol 1994-1995;18:225-8. Crossref

5. Harris EJ, Schimka KE, Carlson RM. Complex regional pain syndrome of the pediatric lower extremity: a retrospective review. J Am Podiatr Med Assoc 2012;102:99-104. Crossref

6. Naleschinski D, Baron R. Complex regional pain syndrome type I: neuropathic or not? Curr Pain Headache Rep 2010;14:196202. Crossref

7. Kahn P. Juvenile idiopathic arthritis: an update for the clinician. Bull NYU Hosp Jt Dis 2012;70:152-66.

8. Cassidy TJ, Patty RE. Juvenile Rheumatoid Arthritis. Textbook of Pediatric Rheumatology. Third Ed. WB. Saunders 1995:133223.

9. Beukelman T, Patkar NM, Saag KG, Tolleson-Rinehart S, Cron RQ, DeWitt EM, et al. 2011 American College of Rheumatology recommendations for the treatment of juvenile idiopathic arthritis: initiation and safety monitoring of therapeutic agents for the treatment of arthritis and systemic features. Arthritis Care Res (Hoboken) 2011;63:465-82. Crossref

10. Weiss PF. Diagnosis and treatment of enthesitis-related arthritis. Adolesc Health Med Ther 2012;2012:67-74. Crossref

11. Baysal Ö, Altay ZE, Durmuş B, Baysal T. Is it Complex Regional Pain Syndrome Type 1 or Inflammatory Arthritis? Turk J Phys Med Rehab 2011;57:45-50. 